PROCEEDINGS OF THE

AMERICAN MATHEMATICAL SOCIETY

Volume 126, Number 12, December 1998, Pages 3709-3713

S 0002-9939(98)04493-1

\title{
MAXIMAL ENTROPY PERMUTATIONS OF EVEN SIZE
}

\author{
WILLIAM GELLER AND ZHENHUA ZHANG
}

(Communicated by Mary Rees)

\begin{abstract}
The entropy of a permutation is the least topological entropy of any continuous interval map having an invariant set which is shuffled according to the permutation. For each $k$, we identify the maximal entropy permutations of size $2 k$.
\end{abstract}

\section{INTRODUCTION}

A finite union of periodic orbits $p_{1}<p_{2}<\ldots<p_{n-1}<p_{n}$ of a (continuous) interval map $f$ induces a permutation $\theta$ on $\{1,2, \ldots, n\}$ in an obvious way using the order on the interval, so that $f\left(p_{i}\right)=p_{\theta(i)}$ for $1 \leq i \leq n$. A surprising amount of dynamical information about the map $f$ can often be recovered from this single permutation, sometimes called the type of the original invariant set. For example, one can typically infer the existence of many other periodic orbits of various types, and obtain a positive lower bound for the topological entropy of $f$. A number of authors have studied these implications and related aspects of combinatorial dynamics in the last several years; see for example [ALM] and the references cited there.

Define the entropy $h(\theta)$ of a permutation $\theta$ to be the infimum of the topological entropies of all interval maps having an invariant set whose type is the permutation. A maximal entropy $n$-permutation is one whose entropy is largest among all $n$-permutations. Misiurewicz and Nitecki [MN] characterized the asymptotic growth in entropy of the maximal entropy permutations for large $n$ and asked which permutations achieved the maximum. For $n$ odd, this question has been answered ([GT], $[\mathrm{GW}])$; the maximal entropy permutations are cycles in a family shown to be nearly maximal in [MN].

If we define the dual of an $n$-permutation $\theta$ as the permutation $\bar{\theta}$ obtained by reversing orientation, $\bar{\theta}(i)=n+1-\theta(n+1-i)$ for $1 \leq i \leq n$, then it is easy to see that $h(\bar{\theta})=h(\theta)$. For each odd $n$, there is a single dual pair of cyclic maximal entropy $n$-permutations.

Here we resolve the question for $n$ even, where the situation turns out to be somewhat different. We show that for each even $n>2$ there are exactly two maximal entropy $n$-permutations $\theta_{n}$ and $\theta_{n}^{\prime}$, which are self-dual and composed variously of 4-cycles, 2-cycles, and fixed points. We note that the natural companion question of finding the maximal entropy cycles of even period remains open.

We would like to thank Fiacre OCairbre for his helpful comments on this work.

Received by the editors January 9, 1997.

1991 Mathematics Subject Classification. Primary 58F08, 54H20.

(C) 1998 American Mathematical Society 


\section{Statement}

We write $P_{n}$ for the set of permutations on $\{1,2, \ldots, n\}$. To avoid the trivial case $n=2$, we assume from here on that $n>2$. For $n$ even, let $k=n / 2$ and define $\theta_{n} \in P_{n}$ by

$$
\begin{gathered}
\theta_{n}(k-i)=\left\{\begin{array}{l}
i+1 \quad \text { if } \quad 1 \leq i<k \text { and } i \text { odd; } \\
n-i \quad \text { if } 0 \leq i<k \text { and } i \text { even; }
\end{array}\right. \\
\theta_{n}(k+i)=\left\{\begin{array}{l}
i \text { if } 1 \leq i \leq k \text { and } i \text { odd; } \\
n-i+1 \quad \text { if } 2 \leq i \leq k \text { and } i \text { even. }
\end{array}\right.
\end{gathered}
$$

Similarly, define $\theta_{n}^{\prime} \in P_{n}$ by

$$
\begin{gathered}
\theta_{n}^{\prime}(k-i)=\left\{\begin{array}{cc}
n-i & \text { if } \quad 1 \leq i<k \\
i+1 & \text { if } 0 \leq i<k
\end{array} \text { and } i\right. \text { odd } \\
\theta_{n}^{\prime}(k+i)=\left\{\begin{array}{l}
n-i+1 \quad \text { if } \quad 1 \leq i \leq k \text { and } i \text { odd } \\
i \text { if } 2 \leq i \leq k \text { and } i \text { even. }
\end{array}\right.
\end{gathered}
$$

For example, using cycle notation we have $\theta_{6}=(14)(2)(36)(5), \theta_{6}^{\prime}=(13)(25)(46)$, $\theta_{8}=(1485)(3267)$, and $\theta_{8}^{\prime}=(1584)(2376)$.

It is easily verified that $\theta_{n}$ and $\theta_{n}^{\prime}$ are self-dual, i.e., $\bar{\theta}_{n}=\theta_{n}$ and ${\overline{\theta^{\prime}}}_{n}=\theta_{n}^{\prime}$.

The permutation $\theta_{n}$ decomposes into disjoint 4-cycles if $k=n / 2$ is even, 2-cycles if $k \equiv 1(\bmod 4)$, and 2-cycles together with two fixed points if $k \equiv 3(\bmod 4)$.

Similarly, $\theta_{n}^{\prime}$ is a composition of 4 -cycles if $k$ is even, of 2 -cycles if $k \equiv 3(\bmod 4)$, and of 2 -cycles together with two fixed points if $k \equiv 1(\bmod 4)$.

Our main result is the following.

Theorem 1. For $n$ even, the permutations $\theta_{n}$ and $\theta_{n}^{\prime}$ have maximal entropy in $P_{n}$, and they are the only maximal entropy permutations.

\section{ProOF}

The primitive function $f_{\theta}$ of a permutation $\theta \in P_{n}$ is the piecewise linear interpolation of $\theta$ on the interval $[1, n]$. The induced matrix $M(\theta)$ is the $(n-1) \times(n-1)$ matrix whose $(i, j)$ th entry is 1 if $f_{\theta}([i, i+1])$ contains $[j, j+1]$ and 0 otherwise. It is well known $[\mathrm{BC}]$ that $h(\theta)=h\left(f_{\theta}\right)=\log \lambda$, where $\lambda$ is the spectral radius of $M(\theta)$.

The proof thus involves estimating the spectral radii of various $0-1$ matrices. The main idea, first used in [MN], is to define a certain cone in $\mathbf{R}^{n-1}$ which can be used to carry out the estimates.

We use several lemmas.

Lemma 1. Let $n$ be even, $k=n / 2$, and $A=M\left(\theta_{n}\right)$. Then

$$
A_{i j}=1 \text { iff }\left\{\begin{array}{cl}
k-j+1 \leq i \leq k+j & \text { for odd } j<k \\
k-j \leq i \leq k+j-1 & \text { for even } j<k \\
1 \leq i \leq 2 k-1 & \text { for } j=k \\
j-k \leq i \leq 3 k-j-1 & \text { for odd } j>k \\
j-k+1 \leq i \leq 3 k-j & \text { for even } j>k .
\end{array}\right.
$$


Also, if $A^{\prime}=M\left(\theta_{n}^{\prime}\right)$, then

$$
A_{i j}^{\prime}=1 \text { iff }\left\{\begin{array}{cl}
k-j \leq i \leq k+j-1 & \text { for odd } j<k \\
k-j+1 \leq i \leq k+j & \text { for even } j<k \\
1 \leq i \leq 2 k-1 & \text { for } j=k \\
j-k+1 \leq i \leq 3 k-j & \text { for odd } j>k \\
j-k \leq i \leq 3 k-j-1 & \text { for even } j>k .
\end{array}\right.
$$

The proof is routine; we omit it.

With definitions as above, we immediately get the following corollaries:

Corollary 1. If $k$ is even, then $A=M\left(\theta_{2 k}\right)$ and $A^{\prime}=M\left(\theta_{2 k}^{\prime}\right)$ are symmetric; if $k$ is odd, then $A^{\prime}$ is the transpose of $A$.

We write $M^{(j)}$ for the $j$ th column of the matrix $M$.

Corollary 2. $A$ and $A^{\prime}$ have $j$ th column sum

$$
\left|A^{(j)}\right|=\left|A^{\prime(j)}\right|=\left\{\begin{array}{l}
2 j \quad \text { if } 1 \leq j<k \\
n-1 \text { if } j=k \\
2(n-j) \text { if } k<j \leq n-1 .
\end{array}\right.
$$

Corollary 3. $A$ and $A^{\prime}$ are irreducible.

We say that a square matrix $M$ of size $2 k-1$ is $A$-like if $\min \left\{i: M_{i}^{(j)} \neq 0\right\}$ for each $k<j \leq 2 k-1$ and $\min \left\{i: M_{2 k-i}^{(j)} \neq 0\right\}$ for each $1 \leq j<k$ are odd, and $A^{\prime}$-like if $\min \left\{i: M_{i}^{(j)} \neq 0\right\}$ for each $1 \leq j<k$ and $\min \left\{i: M_{2 k-i}^{(j)} \neq 0\right\}$ for each $k<j \leq 2 k-1$ are odd.

Corollary 4. For $n=2 k$ the matrices $A=M\left(\theta_{n}\right)$ and $A^{\prime}=M\left(\theta_{n}^{\prime}\right)$ are A-like and $A^{\prime}$-like respectively.

Lemma 2. Let $n=2 k$, and let $B=M(\theta)$, where $\theta \in P_{n}$ is entropy maximal among permutations of length $n$. Then $B$ is A-like or $A^{\prime}$-like.

Proof. For $k<j \leq n-1$, define $i^{-}(j):=\min \left\{i: f_{\theta}(i) \geq j+1\right\} \in\{1, \ldots, n\}$. Similarly, for $1 \leq j<k$, define $i_{+}(j):=\max \left\{i: f_{\theta}(i) \leq j\right\} \in\{1, \ldots, n\}$. Since $\theta$ has maximal entropy, $f_{\theta}$ is maximodal (i.e., has $n$ local extrema) and each minimum value of $f_{\theta}$ lies below each maximum value $[\mathrm{MN}]$. Suppose that $f_{\theta}(1)<f_{\theta}(2)$. Then $f_{\theta}(1) \leq k$, since $f_{\theta}$ has each minimum value below every maximum value. So for each $k<j \leq n-1$ we have $i^{-}(j)>1$; hence $i^{-}(j)$ is a maximum since otherwise it would be a minimum, but then we would have $f_{\theta}\left(i^{-}(j)-1\right)>f_{\theta}\left(i^{-}(j)\right) \geq j+1$, contradicting the definition of $i^{-}(j)$. But then maximodality tells us that $i^{-}(j)$ is even.

Similarly, for each $1 \leq j<k, i_{+}(j)$ is a minimum, and therefore maximodality tells us that $i_{+}(j)$ is odd.

This implies that $B$ is $A$-like, since for $k<j \leq n-1$ we have $\min \left\{i: B_{i}^{(j)} \neq\right.$ $0\}=i^{-}(j)-1$ and for $1 \leq j<k$ we have $\min \left\{i: B_{n-i}^{(j)} \neq 0\right\}=n-i_{+}(j)$.

If, on the other hand, $f_{\theta}(1)>f_{\theta}(2)$, we get analogously that $B$ is $A^{\prime}$-like.

Definition 1. A vector $v \in \mathbf{R}^{n-1}=\mathbf{R}^{2 k-1}$ belongs to $\mathcal{P}$ if

1) $v_{j}=v_{n-j}$ for $1 \leq j<k$, and

2) $v_{k} \geq v_{k-1} \geq \cdots \geq v_{2} \geq v_{1} \geq 0$.

Thus $\mathcal{P}$ is a closed (lower dimensional) convex subcone of the positive cone $\mathbf{R}_{+}^{n-1}=\left\{v \in \mathbf{R}^{n-1}: v \geq 0\right\}$. 
Lemma 3. Multiplication by $A=M\left(\theta_{n}\right)$ leaves $\mathcal{P}$ invariant: $\mathcal{P} A \subset \mathcal{P}$.

Proof. Let $v \in \mathcal{P}$ and let $w=v A$. For $1 \leq p \leq k-1$, define

$$
V(p)=\sum_{j=p}^{k-1} v_{j}
$$

and set $V(k)=0, v_{0}=0$. Then applying Lemma 1 gives, for $1 \leq j<n, w_{j}=$ $(v A)_{j}=v A^{(j)}=2 V(|k-j|+1)+v_{k}+v_{|k-j|}$. Thus for $1 \leq j<k$ we have $w_{n-j}=w_{2 k-j}=w_{j}$. Moreover, for $1 \leq j \leq k$,

$$
\begin{aligned}
w_{j}-w_{j-1} & =2[V(k-j+1)-V(k-j+2)]+v_{k-j}-v_{k-j+1} \\
& =v_{k-j+1}+v_{k-j} \geq 0 .
\end{aligned}
$$

Hence $w \in \mathcal{P}$.

Referring again to Lemma 1, we immediately get the following.

Corollary 5. For $n=2 k$ and $v \in \mathcal{P}$ the matrices $A=M\left(\theta_{n}\right)$ and $A^{\prime}=M\left(\theta_{n}^{\prime}\right)$ satisfy $v A=v A^{\prime}$.

Thus $\mathcal{P} A^{\prime}=\mathcal{P} A \subset \mathcal{P}$.

Lemma 4. If $r$ is a (left) Perron-Frobenius eigenvector for $A=M\left(\theta_{n}\right)$, then it lies in the interior of $\mathcal{P}$.

Proof. By Lemma 3 and the uniqueness of the Perron-Frobenius positive eigendirection, $r$ lies in $\mathcal{P}$. We need to exclude the possibility that it lies on the boundary. But this is clear: as in the proof of Lemma 3 we have for $1 \leq j \leq k$ that $r_{j}-r_{j-1}=r_{k-j+1}+r_{k-j}$, which is positive since irreducibility implies the positivity of $r$.

Corollary 6. $A=M\left(\theta_{n}\right)$ and $A=M\left(\theta_{n}^{\prime}\right)$ have the same spectral radius and the same left Perron-Frobenius eigenvectors.

Lemma 5 ([MN]; see also $[\mathrm{GT}])$. If $n=2 k$, and $B=M(\theta)$, where $\theta \in P_{n}$, then $B$ has $j$ th column sum $\left|B^{(j)}\right| \leq \min (2 j, 2(n-j))$ for $1 \leq j \leq n-1$.

Let $n$ be even, $A=M\left(\theta_{n}\right), B=\left(B_{i j}\right)$ be a nonnegative $(n-1) \times(n-1)$ matrix, and $\epsilon>0$. If $B$ is $A$-like, we define the matrix $B^{\epsilon}=\left(B_{i j}^{\epsilon}\right)$ as in [GW] by

$$
B_{i j}^{\epsilon}=B_{i j}+\epsilon\left[1-\prod_{i=1}^{n-1} \delta\left(A_{i j}, B_{i j}\right)\right],
$$

where $\delta(\cdot, \cdot)$ is Kronecker's delta. In other words, $B_{i j}^{\epsilon}=B_{i j}+\epsilon$ if the $j$ th column of $B$ differs from the $j$ th column of $A$, and otherwise $B_{i j}^{\epsilon}=B_{i j}$. Similarly, if $B$ is not $A$-like but is $A^{\prime}$-like, we replace $A$ by $A^{\prime}$ in the definition of $B^{\epsilon}$.

In either case, note that $B^{\epsilon}$ is irreducible since it has a positive entry everywhere $A$ (or everywhere $A^{\prime}$ ) does, and both $A$ and $A^{\prime}$ are irreducible.

Lemma 6. Let $n=2 k$ be even, $A=M\left(\theta_{n}\right)$, and let $A$ (and so also $A^{\prime}=M\left(\theta_{n}^{\prime}\right)$ ) have spectral radius $\lambda$ and Perron-Frobenius (left) eigenvector $r$, so that $r A=\lambda r$. Let $B=M(\theta)$, where $\theta \in P_{n}$ has maximal entropy among permutations of length $n$. Then there exists $\epsilon>0$ sufficiently small so that $r A\left(=r A^{\prime}\right) \geq r B^{\epsilon}$. 
Proof. By Corollary 4 and Lemma 2, we know that $B$ is either $A$-like or $A^{\prime}$-like. Suppose the former is true; a similar argument together with Corollary 5 can be used in the latter case.

As in the proof of Lemma 3 , for $1<j \leq k$ we have

$$
(r A)_{j}=\left(2 \sum_{i=k-j+1}^{k-1} r_{i}\right)+r_{k}+r_{k-j}
$$

(where we set $r_{0}=0$ ) and $(r A)_{1}=r_{k}+r_{k-1}$.

Thus $(r A)_{j}=r A^{(j)}$ is the sum of the $2 j$ largest (i.e., most central) coordinates of $r$. Note that although by the symmetry of $r$ and Lemma 4 there are exactly two choices of these $2 j$ largest coordinates, symmetry implies that they have the same sum. By Lemma 5 , the column $B^{(j)}$ has at most $2 j$ ones. So $(r A)_{j}=r A^{(j)} \geq$ $r B^{(j)}=(r B)_{j}$, with equality only if $B^{(j)}=A^{(j)}$ or $B^{(j)}=A^{(n-j)}=A^{(j)}$. But the latter case is ruled out by our assumption that $B$ is $A$-like.

The situation is similar for $j>k$.

Proof of Theorem 1. Let $\theta \in P_{n}$ have maximal entropy among all permutations of length $n$. By Lemma 2, $B=M(\theta)$ is $A$-like or $A^{\prime}$-like; suppose that $B$ is $A$-like. We will show that $h\left(\theta_{n}\right) \geq h(\theta)$, with equality only if $\theta=\theta_{n}$. If $B$ is $A^{\prime}$-like, a similar argument shows that $h\left(\theta_{n}^{\prime}\right)\left(=h\left(\theta_{n}\right)\right) \geq h(\theta)$, with equality only if $\theta=\theta_{n}^{\prime}$.

Let $r$ be a (left) Perron-Frobenius eigenvector for $A=M\left(\theta_{n}\right)$. Let $\lambda$ be the spectral radius of $A$ and $\mu$ the spectral radius of $B$. By Lemma 6 , there exists $\epsilon>0$ sufficiently small so that $r A \geq r B^{\epsilon}$. Now let $\mu_{\epsilon}$ be the spectral radius of $B^{\epsilon}$, and let $s_{\epsilon}$ be a right Perron-Frobenius eigenvector: $B^{\epsilon} s_{\epsilon}=\mu_{\epsilon} s_{\epsilon}$. Then

$$
\lambda\left(r, s_{\epsilon}\right)=\left(\lambda r, s_{\epsilon}\right)=\left(r A, s_{\epsilon}\right) \geq\left(r B^{\epsilon}, s_{\epsilon}\right)=\left(r, B^{\epsilon} s_{\epsilon}\right)=\mu_{\epsilon}\left(r, s_{\epsilon}\right) .
$$

This implies that $\lambda \geq \mu_{\epsilon}$, since $r$ and $s_{\epsilon}$ are positive by Perron-Frobenius and the irreducibility of $A$ and $B^{\epsilon}$. So we have $h\left(\theta_{n}\right)=\log \lambda \geq \log \mu_{\epsilon} \geq \log \mu=h(\theta)$, since $B^{\epsilon} \geq B$.

Furthermore, the latter inequality here is strict (again invoking the irreducibility of $B^{\epsilon}$ ) unless $B^{\epsilon}=B=A$, i.e., unless $\theta=\theta_{n}$.

\section{REFERENCES}

[ALM] L. Alseda, J. Llibre and M. Misiurewicz, Combinatorial dynamics and entropy in dimension one, Advanced Series in Nonlinear Dynamics, vol. 5, World Scientific, Singapore, 1993. MR 95j:58042

[BC] L. Block and W. A. Coppel, Dynamics in one dimension, Lecture Notes in Math., vol. 1513, Springer-Verlag, Berlin and New York, 1992. MR 93g:58091

[GT] W. Geller and J. Tolosa, Maximal entropy odd orbit types, Trans. Amer. Math. Soc. 329 (1992), 161-171. MR 92e:58163

[GW] W. Geller and B. Weiss, Uniqueneness of maximal entropy odd orbit types, Proc. Amer. Math. Soc. 123 (1995), 1917-1922. MR 95g:58172

[MN] M. Misiurewicz and Z. Nitecki, Combinatorial patterns for maps of the interval, Mem. Amer. Math. Soc. 94 (1991), no. 456. MR 92h:58105

Department of Mathematical Sciences, Indiana University-Purdue University IndianApolis, 402 N. Blackford St., Indianapolis, Indiana 46202

E-mail address: wgeller@math.iupui.edu

Department of Mathematics, Tufts University, Medford, Massachusetts 02155

E-mail address: zzhang@diamond.tufts.edu 